\title{
Supercontinuum generation and beatnote detection using ultrafast VECSEL seed oscillators
}

Robert Rockmore, Caleb W. Baker, Alexandre Laurain, Tsung-Han Wu, R. Jason Jones, et al.

Robert Rockmore, Caleb W. Baker, Alexandre Laurain, Tsung-Han Wu, R. Jason Jones, Jerome V. Moloney, "Supercontinuum generation and beatnote detection using ultrafast VECSEL seed oscillators," Proc. SPIE 10515, Vertical External Cavity Surface Emitting Lasers (VECSELs) VIII, 105150M (15 February 2018); doi: 10.1117/12.2291079 


\title{
Supercontinuum generation and beatnote detection using ultrafast VECSEL seed oscillators
}

\author{
Robert Rockmore, Caleb W. Baker, Alexandre Laurain, Tsung-Han Wu, R. Jason Jones, \\ Jerome V. Moloney \\ College of Optical Sciences, The University of Arizona, 1630 E University Blvd, Tucson, AZ, \\ US 85719;
}

\begin{abstract}
We present preliminary results showing the potential of VECSEL technology for the generation of high power coherent supercontinuum. Among these results, we demonstrate a stable output power of $16 \mathrm{~W}$ with a pulse duration of $71 \mathrm{fs}$ and a repetition rate of $1.7 \mathrm{GHz}$ from a VECSEL oscillator and Ytterbium fiber amplifier. This system was used to generate a coherent supercontinuum averaging $3 \mathrm{~W}$ of power using a highly nonlinear photonic crystal fiber. In addition, we discuss the possible methods for the detection and stabilization of the carrier offset frequency. The beatnote between a VECSEL seeded supercontinuum and an external CW laser reveals a relatively stable signal, well above the detection noise. A discussion about system design considerations for noise reduction and increased offset frequency stability is also included.
\end{abstract}

Keywords: VECSELs, frequency combs, nonlinear broadening, supercontinuum generation

\section{INTRODUCTION}

Vertical external cavity surface emitting lasers (VECSELs) can produce ultrashort pulses at high repetition rates in the $\mathrm{GHz}$ regime, and up to $100 \mathrm{GHz}$ by intgrating the saturable absorber into the gain chip. ${ }^{1}$ Other laser technologies utilizing passive modelocking, such as fiber lasers and solid state lasers have trouble reaching these $\mathrm{GHz}$ repetition rates because of the necessary short cavity length and q switching instabilities. ${ }^{2}$ These mode locked laser sources are attractive for use as $\mathrm{GHz}$ frequency combs for precision spectroscopy, because they have more power per individual comb line when compared to lower repetition rate combs, and have larger spacing between these lines. A stable frequency comb requires locking of the carrier envelope offset frequency (CEO). In the time domain, locking the carrier envelope offset fixes the relative phase of the electric field relative to the pulse envelope. In the frequency domain, it corresponds to the absolute offset from zero frequency of the optical comb if it were extended to this zero. The traditional $\mathrm{f}-2 \mathrm{f}$ method for detecting the CEO offset for stabilization requires high peak power to generate an octave spanning supercontinuum in a nonlinear medium such as a photonic crystal fiber, which is difficult to achieve directly from a VECSEL source ${ }^{3}{ }^{4}$ Highly nonlinear waveguides can also be used to perform $\mathrm{f}-2 \mathrm{f}$ measurements directly from a VECSEL, ${ }^{5}$ but output power is limited. Amplifying the VECSEL output with a fiber amplifier is one potential method to overcome this difficulty, and allows nonlinear media such as photonic crystal based nonlinear fibers to be used to detect the offset frequency from a modelocked VECSEL source. ${ }^{6}$ However, for effective stabilization of the offset frequency, a strong $\mathrm{f}-2 \mathrm{f}$ signal is required.

In this proceeding, we discuss a VECSEL based fiber amplified system that produces pulses as short as 71 femtoseconds with over 16 watts of total power. This system was used to produce a multi-watt, octave spanning supercontinuum that can potentially enable higher signal to noise ratio for an f-2f CEO measurement. This wide, high power supercontinuum is also attractive for a variety of other applications, such as nonlinear microscopy for medical imaging. Through optical filtering and pulse shaping, specific wavelength bands of the supercontinuum can be used for different imaging modalities from one laser source. ${ }^{7}$

Further author information:(Send correspondence to R. Rockmore)

R. Rockmore: E-mail: Rockmore@optics.arizona.edu

Vertical External Cavity Surface Emitting Lasers (VECSELs) VIII, edited by

Juan L. Chilla, Proc. of SPIE Vol. 10515, 105150M - (c) 2018 SPIE

CCC code: $0277-786 X / 18 / \$ 18 \cdot$ doi: $10.1117 / 12.2291079$

Proc. of SPIE Vol. 10515 105150M-1 


\section{EXPERIMENTAL SETUP}

\subsection{VECSEL system design}

The experimental setup utilized for the supercontinuum generation consisted of one of two distinct passively modelocked VECSEL cavity designs, which then were used to seed a two stage fiber amplifier for power scaling and pulse shortening. The first of these two VECSEL designs was the double-fold cavity introduced in ref, ${ }^{8}$ which employs a single gain chip and semiconductor saturable absorber mirror (SESAM). This cavity design features four passes on the gain and one pass on the SESAM, in contrast to the typical two gain passes in a standard V cavity design with a curved output coupler and the SESAM as an end mirror. This multi-pass design allows for lower repetition rates than a typical V cavity without multi-pulsing or other instabilities forming. The VECSEL used here consisted of a resonant periodic gain (RPG) structure VECSEL chip emitting 500 fs pulses centered at $1020 \mathrm{~nm}$ with a repetition rate of $390 \mathrm{MHz}$ and and operating with average output power of approximately 150 $\mathrm{mW}$. The gain chip used in this device consisted of a resonant periodic gain (RPG) active region with a single quantum well under each antinode of the electric field. This chip was coated with a single layer SiN coating for dispersion management.

The second VECSEL system used was a traditional V cavity with a semiconductor saturable absorber mirror (SESAM) as the end mirror and a curved $10 \mathrm{~cm} 1 \%$ output coupler. The VECSEL gain chip was designed for modelocked operation based on the simulations introduced in ref. ${ }^{9}$ This chip consisted of a hybrid metalsemiconductor distributed Bragg reflector and an active region consisting of 12 quantum wells arranged non uniformly under 4 antinodes of the electric field. The surface of the chip was coated with a $\mathrm{Ta}_{2} \mathrm{O}_{5} / \mathrm{SiO}_{2}$ bi-layer anti reflective coating to obtain a flat GDD close to zero around the lasing wavelength of $1040 \mathrm{~nm}$. This laser produced pulses approximately 250 femtoseconds in duration with a repetition rate of $1.7 \mathrm{GHz}$. Average output power was between 70 and $80 \mathrm{~mW}$ for single pulse operation. The combination of lower average power and higher repetition rate of this VECSEL system gives lower peak power than the F cavity based setup despite the shorter pulse duration.

Both of these VECSEL systems were optically pumped by an $808 \mathrm{~nm}$ diode fiber coupled diode laser, and VECSEL and SESAM temperatures were controlled by thermoelectric coolers for tuning the VECSEL performance. In both cases a plastic enclosure was placed around the free running VECSEL system in order to isolate from external noise sources. An optical isolator was placed after the output of the VECSEL to isolate it from any back reflections from downstream optical components.

\subsection{Amplifier system design}

The fiber amplification scheme consisted of low power preamplifier followed by a power amplifier. In both cases the gain fiber was a Ytterbium doped double clad polarization maintaining fiber (Nufern PLMA-YDF-10/125) with lengths of $2.5 \mathrm{~m}$ and $7 \mathrm{~m}$ for the preamp and power amp, respectively. This fiber features an $11 \mu \mathrm{m}$ core diameter and was pumped by $915 \mathrm{~nm}$ fiber coupled diode lasers for both the preamplifier and power amplifier. The first amplifier stage was pumped such that it produced an output of approximately $500 \mathrm{~mW}$ in both cases for optimal seeding of the power amplifier. The preamplifier stage is not strictly necessary, but was used to increase the reliability of the setup and to ensure adequate seeding of the high power amplifier under high power conditions to prevent damage. The preamplifier was followed by a pair of transmission gratings in a double pass configuration to adjust the chirp of the pulses to produce maximum nonlinear broadening in the power amplifier stage.

The power amplifier was designed for nonlinear spectral broadening and pulse compression based on systems shown in ref ${ }^{10}$ and ref. ${ }^{11}$ By adjusting the spacing of the pre-chirp gratings, the pulses can be optimized to produce nonlinear broadening in the power amplifier stage that can be easily compressed afterwards using a second grating pair to achieve a pulse duration below $100 \mathrm{fs}$. After the power amplifier, power levels were approaching $17 \mathrm{~W}$ for both the F-cavity and V-cavity systems. Both amplifiers had angle-cleaved facets, with an endcap on the output facet consisting of a 200-500 $\mu \mathrm{m}$ piece of glass spliced into the end to prevent reflections from within the amplifier from coupling back into the fiber. Figure 1 shows the basic layout of the system for the $\mathrm{V}$-cavity arrangement including the photonic crystal fiber discussed in the next section. 


\subsection{Pulse compression and performance}

After the nonlinear amplification, the optical bandwidth was broadened significantly. This spectrum was able to produce pulses of $71 \mathrm{fs}$ after grating compression with 16.45 watts of output power. Figure 2 shows the spectral and temporal characteristics of the compressed pulses measured with a FROG system. These measurements indicate good pulse shape and the phase shows low second and third order dispersion, signs of an adequate pulse compression. The combination of short pulse duration and high power make this system attractive as a high repetition rate source for nonlinear applications.

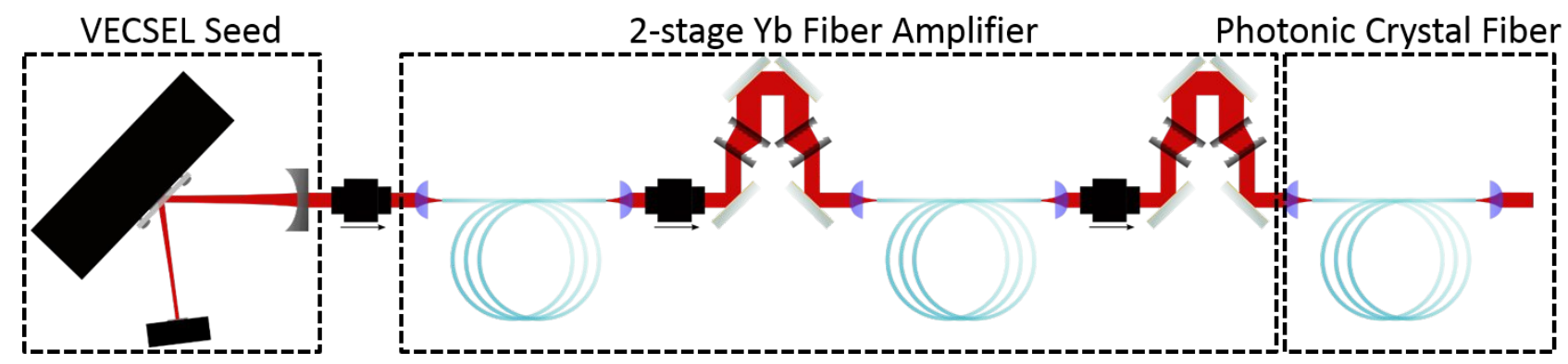

Figure 1. Overview of setup, featuring (from left to right): V cavity VECSEL seed, fiber preamplifier, fiber power amplifier, and photonic crystal fiber. Also pictured are optical isolators between each stage and grating compressors after the preamplifier and power amplifier stages.

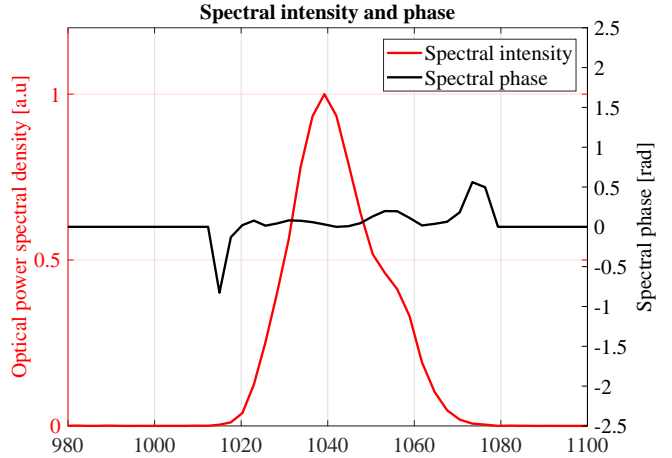

(a)

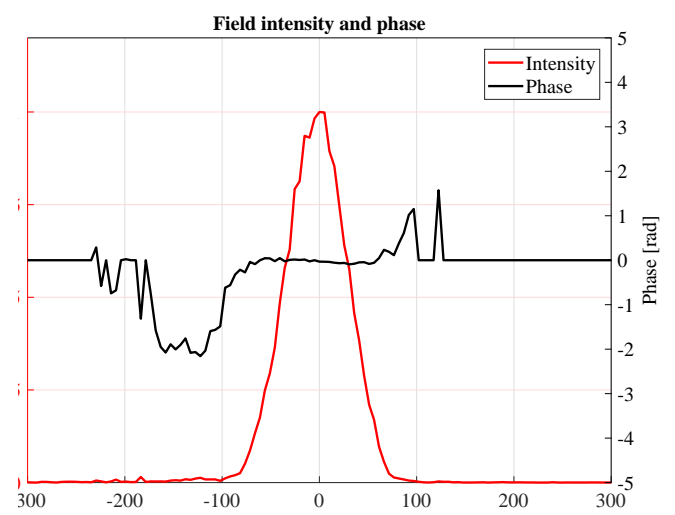

(b)

Figure 2. FROG traces of spectral intensity and phase (a) and Field intensity and phase in time (b) for 70 fs compressed pulse after power amplifier. These results were obtained with the $1.7 \mathrm{GHz} \mathrm{V}$ cavity seed.

\section{BROADENING AND BEAT NOTE DETECTION}

Following the final compression stage was one of two nonlinear photonic crystal fibers designed to produce a much broader spectrum than what could be achieved out of the amplifier. The first fiber was 2 meters of Thorlabs LMA-PM-5 fiber with a 5 micron core, a mode field diameter of $4.4 \mu \mathrm{m}$, and a zero dispersion wavelength of $1040 \mathrm{~nm}$. The second fiber was 1.5 meters of NKT Photonics SC-3.7-975, which has a $3.7 \mu \mathrm{m}$ core, $3.2 \mu \mathrm{m}$ mode field diameter, and zero dispersion wavelength of $975 \mathrm{~nm}$. The Thorlabs fiber was used with both the F-cavity arrangement and the V-cavity arrangement. The NKT Photonics fiber was only used with the V-cavity setup. The nonlinear coefficient of the Thorlabs fiber around the zero dispersion wavelength of 1040nm was approximately $\gamma_{1040}=9 \frac{1}{W * k m}$, and the value for the NKT photonics fiber was $\gamma_{975}=18 \frac{1}{W * k m}$.

In order to prevent damage to the nonlinear fiber, the incident power was attenuated using a polarizing filter consisting of a half wave plate and a polarizing beamsplitter. In this way, power incident on the PCF could be tuned independently from the amplifier. 


\subsection{F-cavity broadening results}

The first results were obtained with the 2 meters of Thorlabs PCF and approximately $1 \mathrm{~W}$ of input power, and are shown in Fig 3. The output power in this case was $300-400 \mathrm{~mW}$ and the broadened spectrum extended from $800 \mathrm{~nm}$ to just below $1500 \mathrm{~nm}$. At input powers above $1 \mathrm{~W}$, thermal issues reduced coupling efficiency and eventually led to damage at around $2 \mathrm{~W}$. The lower $390 \mathrm{MHz}$ repetition rate of the F-cavity allows for higher peak power for a given incident average power when compared to higher repetition rate $\mathrm{V}$ cavity design. Because the thermal damage is dependent on the average power, a lower repetition rate oscillator allows us to have higher maximum peak power coupled into the PCF before thermal damage occurs.

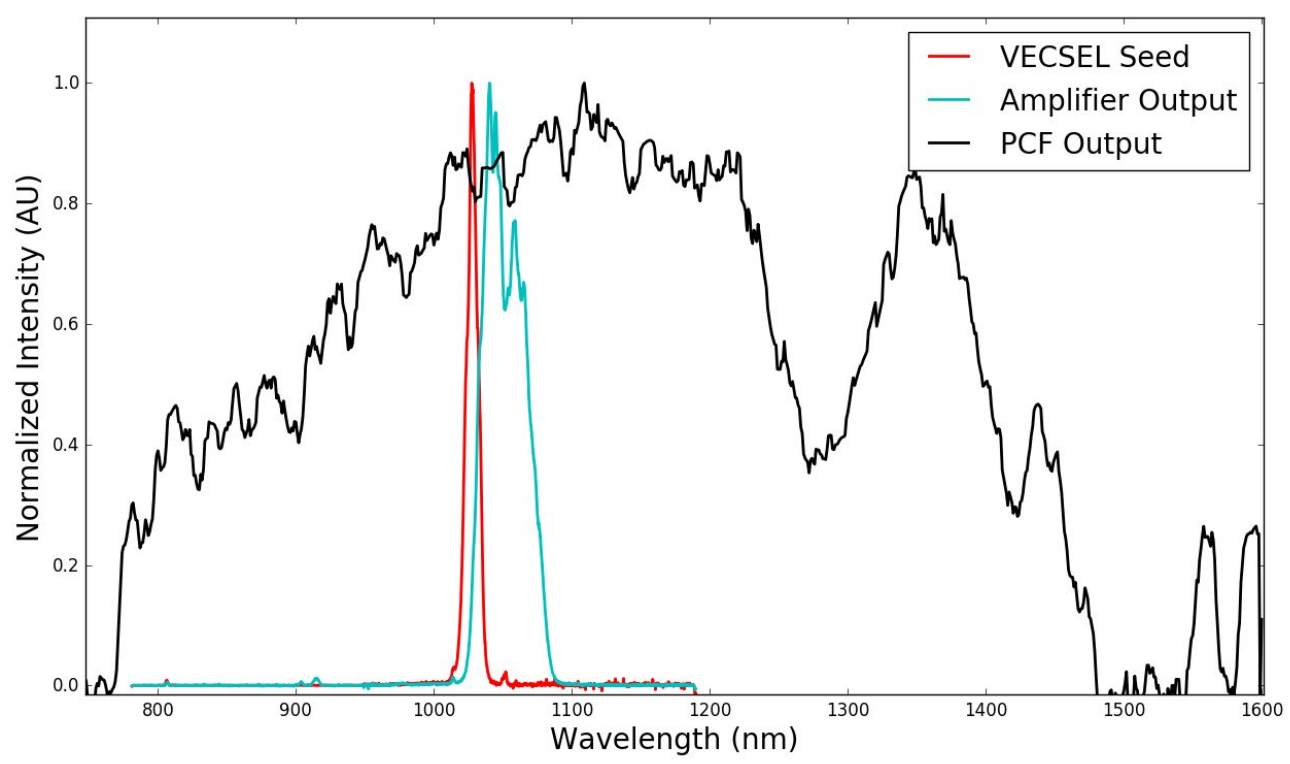

Figure 3. Supercontinuum generated with $390 \mathrm{MHz}$ repetition rate double folded "F-cavity," in comparison with spectrum directly from the VECSEL seed and spectrum after the nonlinear broadening in the power amplifier. This broadening spectrum was obtained with 2 meters of Thorlabs LMA-PM-5 fiber.

\section{$3.2 \mathrm{~V}$ cavity broadening results}

The system was then rebuilt in the V-cavity configuration with the multi quantum well chip and optimized for short duration, single pulsed operation in order to produce a better final spectrum after broadening in the PCF. This system had a lasing wavelength centered closer to $1040 \mathrm{~nm}$, which suited the ytterbium gain better than the $1020 \mathrm{~nm}$ F-cavity system. In order to increase the power handling capability of the PCF, a short endcap was attached to the end. This endcap consisted of a length of multimode fiber a few hundred microns in length directly spliced to the end of the photonic crystal fiber. This allows the high NA coupling lens to focus the light to its highest irradiance within the bulk glass medium instead of at the air/glass interface, increasing the damage threshold. Utilizing this technique, the maximum input power before damage was increased to approximately $9 \mathrm{~W}$. However, the higher repetition rate of the V-cavity results in peak power that is approximately 4.5 times lower than that with the F-cavity seed for a given average power. Because of this, the spectrum obtained with this fiber at an input power of $8 \mathrm{~W}$ only spans from $800 \mathrm{~nm}$ to almost $1400 \mathrm{~nm}$ with an output power of 3 $\mathrm{W}$. This spectrum is shown in 4. Although this spectrum is not quite sufficient for an $\mathrm{f}-2 \mathrm{f}$ measurement, it could be utilized for multimodal microscopy applications or other non-linear applications requiring a coherent multi-spectral source.

After reaching the input power limit with the Thorlabs fiber we switched to the NKT Photonics fiber and endcapped the input facet as before. The maximum input power used on this fiber was $6 \mathrm{~W}$, producing the 


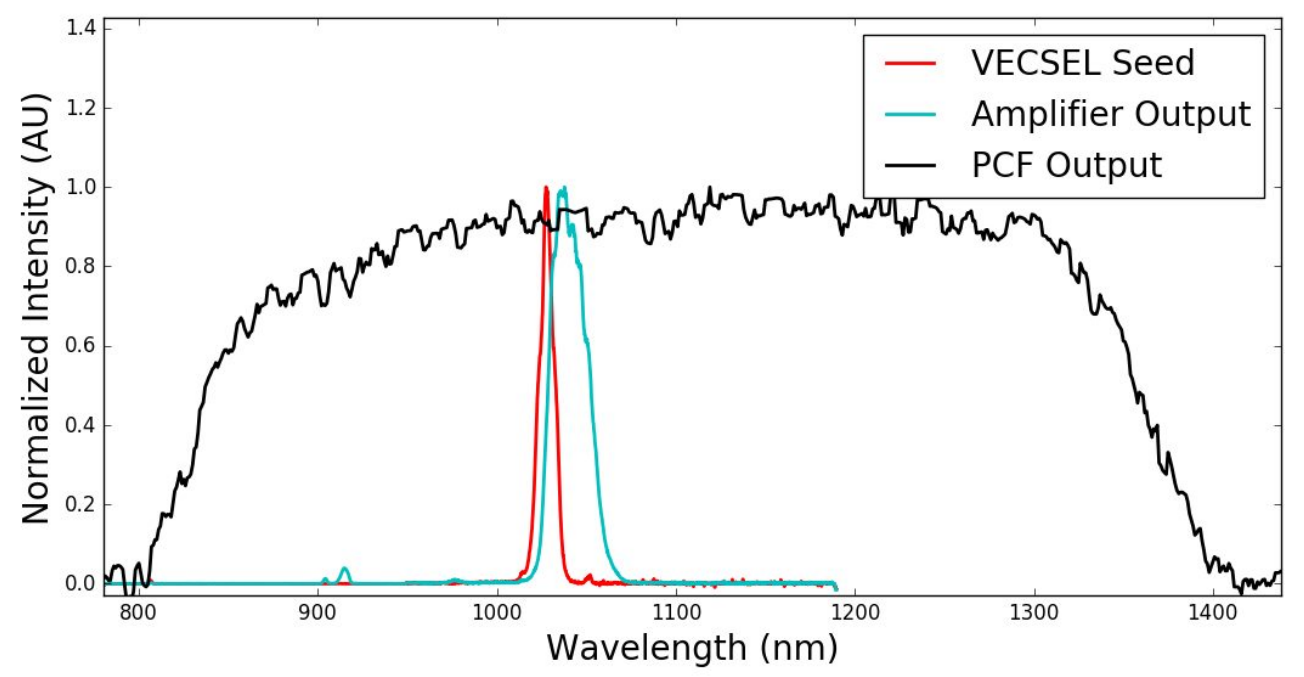

Figure 4. V cavity spectrum directly out of the oscillator, after the amplifier, and after broadening through 2 meters of Thorlabs PCF (with endcap on PCF).

spectrum shown in figure 5 and resulting in $2 \mathrm{~W}$ of output power. This spectrum spans an entire octave from 700-1400 nm, making it attractive for doubling with periodically-poled lithium niobate (PPLN) to detect the offset frequency in an f-2f measurement for CEO stabilization.

A future investigation could utilize the lower repetition rate F-cavity oscillator in conjunction with the coupling and power handling improvements yielded by the PCF endcap to produce even further broadening with a $6 \mathrm{~W}$ average power level.

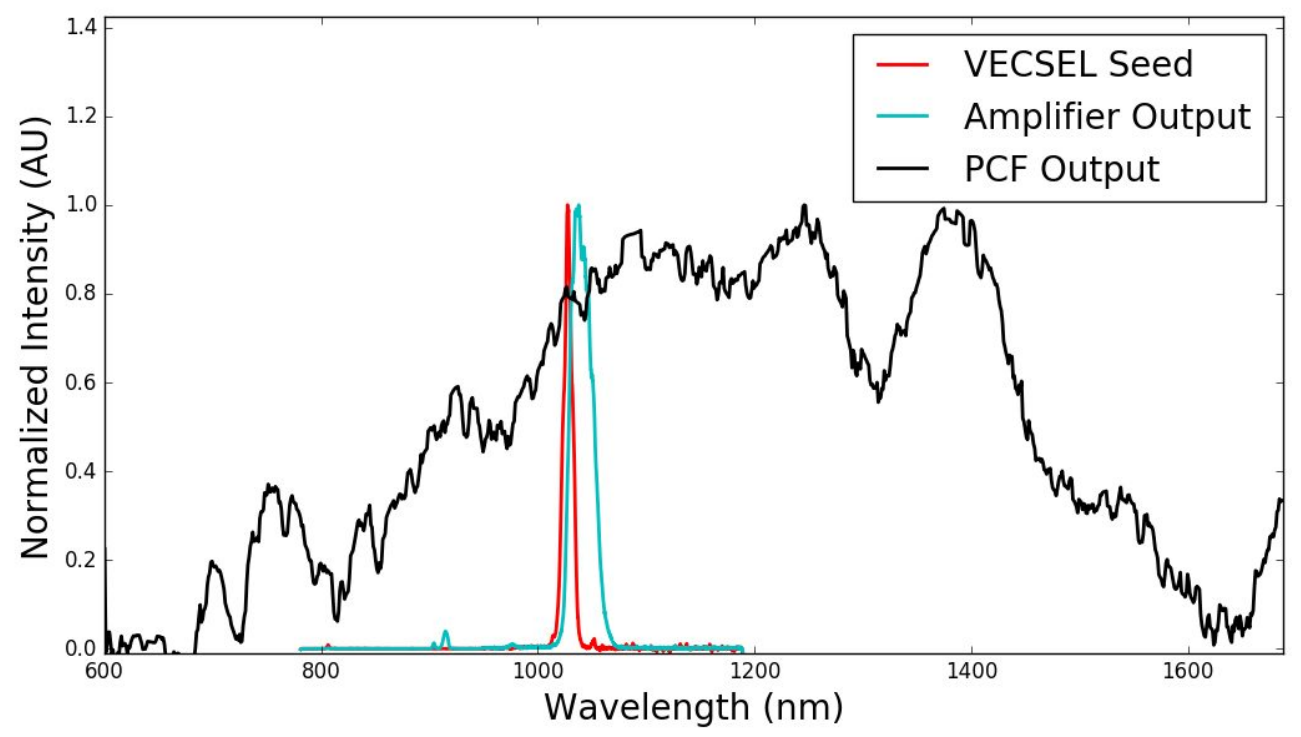

Figure 5. V cavity spectra with end-capped NKT Photonics Fiber with $6 \mathrm{~W}$ input power at PCF. 


\subsection{Beat note detection}

In order to verify the coherence of this octave spanning supercontinuum on the short end of the wavelength spectrum, a single frequency $780 \mathrm{~nm}$ Vescent DBR laser was combined with the output of the PCF using a beamsplitter. This combined spectrum was then directed to a grating, where the $780 \mathrm{~nm}$ component was isolated with an aperture and focused onto a fast photodiode. A beat note between the two beams was observed with a signal approximately $25 \mathrm{~dB}$ above the noise floor with a $1 \mathrm{MHz}$ resolution bandwidth. This beat note confirms that the supercontinuum is coherent at $780 \mathrm{~nm}$. Ongoing efforts to better isolate the VECSEL system from the environment and reduce amplitude noise of the pump laser in order to stabilize this system further are currently underway. The higher power of this supercontinuum comapared to previous results may allow for better signal to noise ratio for the f-2f beat note and improving the quality of the CEO frequency lock. ${ }^{6}$

\section{SUMMARY}

We developed an amplified VECSEL based system capable of producing $71 \mathrm{fs}$ pulses with a total average power of over $16 \mathrm{~W}$ and a repetition rate of $1.7 \mathrm{GHz}$. This system utilized a nonlinear amplification and compression technique in order to produce short pulses, which were then coupled into two different photonic crystal fibers for nonlinear broadening with input powers of up to 8 watts. This resulted in a multi watt, octave spanning supercontinuum that can be used for $\mathrm{f}-2 \mathrm{f} \mathrm{CEO}$ frequency detection for frequency comb stabilization, or for various other applications that benefit from a wide, high average power spectrum such as nonlinear imaging techniques.

\section{ACKNOWLEDGMENTS}

We would like to thank Wolfgang Stolz and Antje Ruiz Perez at Philipps Universität, Marburg for growing our VECSEL structures, and Ganesh Balakrishnan and Sadhvikas Addamane at the University of New Mexico for growing our SESAMs. This material is based upon work supported by the Air Force Office of Scientific Research under award ID FA9550-17-1-0246 and FA9550-14-1-0062.

\section{REFERENCES}

[1] Mangold, M., Zaugg, C. A., Link, S. M., Golling, M., Tilma, B. W., and Keller, U., "Pulse repetition rate scaling from 5 to $100 \mathrm{GHz}$ with a high-power semiconductor disk laser," Optics Express 22, 6099-6107 (Mar. 2014).

[2] Hnninger, C., Paschotta, R., Morier-Genoud, F., Moser, M., and Keller, U., "Q-switching stability limits of continuous-wave passive mode locking," JOSA B 16, 46-56 (Jan. 1999).

[3] Jones, D. J., Diddams, S. A., Ranka, J. K., Stentz, A., Windeler, R. S., Hall, J. L., and Cundiff, S. T., "Carrier-Envelope Phase Control of Femtosecond Mode-Locked Lasers and Direct Optical Frequency Synthesis," Science 288, 635-639 (Apr. 2000).

[4] Dudley, J. M., Genty, G., and Coen, S., "Supercontinuum generation in photonic crystal fiber," Reviews of Modern Physics 78, 1135-1184 (Oct. 2006).

[5] Waldburger, D., Mayer, A. S., Alfieri, C. G. E., Johnson, A. R., Johnson, A. R., Ji, X., Ji, X., Klenner, A., Okawachi, Y., Lipson, M., Gaeta, A. L., and Keller, U., "Self-referenced CEO Frequency Detection of a Semiconductor Disk Laser using a Silicon Nitride Waveguide," in [Laser Congress 2017 (ASSL, LAC) (2017), paper ATu6A.3], ATu6A.3, Optical Society of America (Oct. 2017).

[6] M. Mangold, C. A. Zaugg, Alexander Klenner, A. S. Mayer, S. M. Link, F. Emaury, M. Golling, Emilio Gini, C. J. Saraceno, B. W. Tilma, and U. Keller, "Carrier-envelope-offset frequency detection of an ultrafast VECSEL," Proc.SPIE 9349, 9349 - 9349 - 7 (2015).

[7] Liu, Y., Tu, H., Benalcazar, W. A., Chaney, E. J., and Boppart, S. A., "Multimodal Nonlinear Microscopy by Shaping a Fiber Supercontinuum From 900 to 1160 nm," IEEE Journal of Selected Topics in Quantum Electronics 18, 1209-1214 (May 2012).

[8] Baker, C. W., Scheller, M., Laurain, A., Ruiz-Perez, A., Stolz, W., Addamane, S., Balakrishnan, G., Koch, S. W., Jones, R. J., and Moloney, J. V., "Multi-Angle VECSEL Cavities for Dispersion Control and PeakPower Scaling," IEEE Photonics Technology Letters 29, 326-329 (Feb. 2017). 
[9] Kilen, I., Koch, S. W., Hader, J., and Moloney, J. V., "Non-equilibrium ultrashort pulse generation strategies in VECSELs," Optica 4, 412-417 (Apr. 2017).

[10] Wang, S., Liu, B., Gu, C., Song, Y., Qian, C., Hu, M., Chai, L., and Wang, C., "Self-similar evolution in a short fiber amplifier through nonlinear pulse preshaping," Optics Letters 38, 296-298 (Feb. 2013).

[11] Liu, W., Schimpf, D. N., Eidam, T., Limpert, J., Tnnermann, A., Krtner, F. X., and Chang, G., "Pre-chirp managed nonlinear amplification in fibers delivering 100 W, 60 fs pulses," Optics Letters 40, 151-154 (Jan. 2015). 